\title{
Manual Lymphatic Drainage after Subepidermal Tracer Injection Optimizes Results of Sentinel Lymph Node Labeling in Primary Breast Cancer
}

\author{
Martin Sillem $^{a}$ Urban Bromberger $^{b}$ Barbara Heitzelmann ${ }^{c} \quad$ Wolfgang J. Brauer $^{b}$ \\ Martin Werner ${ }^{\mathrm{d}}$ Sylvia Timme ${ }^{\mathrm{d}}$ \\ a Praxisklinik am Rosengarten, Mannheim, Germany; \\ ${ }^{b}$ Department of Radiology and Nuclear Medicine, Kreiskrankenhaus Emmendingen, Emmendingen, Germany; \\ ${ }^{c}$ Department of Obstetrics and Gynecology, Kreiskrankenhaus Emmendingen, Emmendingen, Germany; \\ ${ }^{d}$ Institute of Clinical Pathology, University Hospital Freiburg, Freiburg i.Br., Germany
}

\section{Keywords}

Breast cancer - Sentinel node $\cdot$ Manual lymphatic drainage

\section{Summary}

Introduction: Our aim was to assess the practicability and reliability of a novel labeling regime for axillary sentinel lymph nodes (SLNs) in early breast cancer. Methods: 362 patients with early breast cancer (bilateral in 9 cases, giving a total of 371 cases) underwent intradermal radio tracer injection with simultaneous manual lymphatic drainage. SLN biopsy was performed within $24 \mathrm{~h}$. For retrospective analysis, data were extracted from patient's records. Results: At least 1 SLN was detected intraoperatively in 369 cases $(99.5 \%$, range $1-9$ nodes). This node was metastatic in 88 and unaffected in 281 cases. Coincidentally removed but unlabeled lymph nodes were affected in 3 cases in which the SLN was unaffected $(3 / 153=2 \%)$. In all cases, on histological evaluation, tissue removed as SLN contained lymph nodes. After a period of 69.5 months (median 1.7-115.8 months), no axillary recurrences were observed in 213 patients. Conclusion: Manual lymphatic drainage is a simple technique that leads to an extremely high pick-up rate of axillary SLNs after subepidermal radio tracer injection. If unaffected, this node correctly predicts nodal-negative disease in $98 \%$ of cases studied.

(c) 2015 S. Karger GmbH, Freiburg

\section{Introduction}

Axillary lymph node involvement remains a principal factor when making clinical decisions in primary breast cancer [1]. However, formal dissection of the axilla entails a high rate of late morbidity, in particular lymph edema, which can occur in $19-47 \%$ of cases [2]. Following the publication of the first large series on sentinel lymph node biopsy (SLNB) in the surgical staging of primary breast cancer, SLNB has found rapid and wide acceptance [3]. Lymph edema in patients undergoing SLNB only is rare [4].

Despite this rapid acceptance, there is still a considerable heterogeneity with respect to the details of the technique. The American College of Breast Surgeons recommends a combination of dye and radioactive labeling, with omission of dye labeling only in experienced centers. [5]. The American Society of Clinical Oncology gives no additional technical details but recommends an $85 \%$ identification rate and a $5 \%$ false-negative rate as minimal standard [6].

Various methods have been proposed for labeling SLNs, and inconsistent results have been reported with respect to retrieval rates. Intraoperative injection of dye is easy to perform and needs no equipment. Retrieval rates have been reported to range from $88.5 \%$ to $97.5 \%[7,8]$. Radiopharmaceutical labeling yields better results with positive identification of the labeled node in $97.2 \%$ of cases. A combination of both methods has also been described with retrieval rates of $98.7 \%$ [9].

Several large multi-center studies have analyzed false-negative rates, i.e. the frequency of metastatic lymph nodes despite unaffected SLNs, defined as the number of patients with negative SLN and at least 1 positive non-SLN, divided by the total number of

\section{KARGER




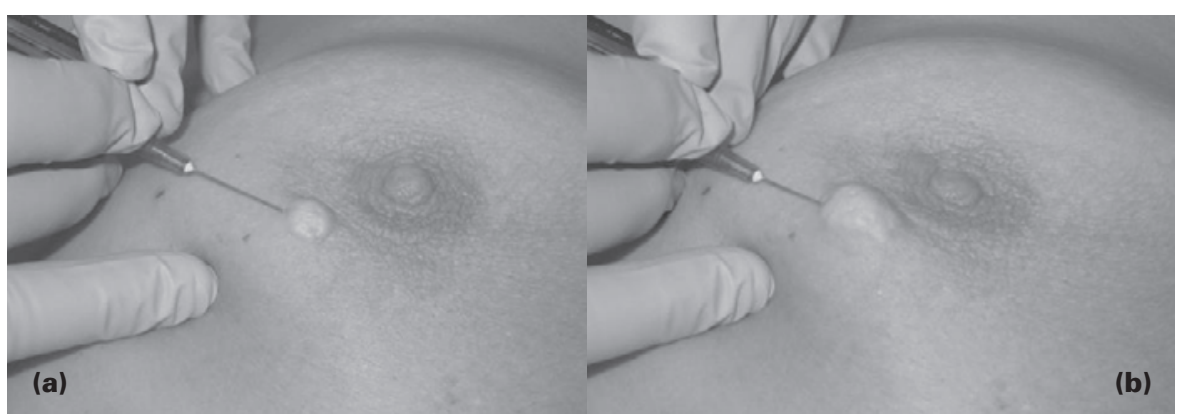

Fig. 1. Intradermal injection technique. a, b Creating the intradermal wheal. c, d Manual lymphatic drainage, using standing circles technique for 2 min: both hands exert increasing pressure while rotating towards the regional lymph nodes and decreasing pressure on the way back. Rubbing the skin is avoided.

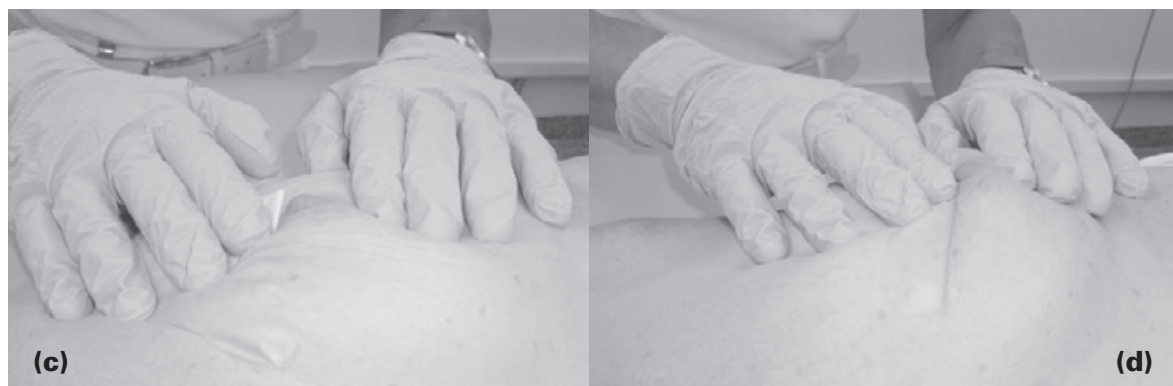

Table 1. Patient characteristics

patients with positive axillary lymph nodes [10]. Chagpar et al. [7] analyzed 3,691cases and compared different injection sites. A combination of peritumoral dye and intradermal radioactive colloid injection resulted in a retrieval rate of $97.4 \%$ and a false-negative rate of $8.3 \%$. In the NSABP-B32 study in which the protocol required injection of both dye and radioactive tracer around the tumor, a retrieval rate of $96.9 \%$ and a false-negative rate $9.5 \%$ was observed in 4,994 patients [10].
Here, we describe and evaluate a standardized regime of subepidermal radioactive tracer injection that takes into consideration the physiology of lymphatic transport using a standard lymph draining procedure. The method gives results that are superior to those previously reported without the need for dye injection.

\section{Patients and Methods}

\section{Patient Characteristics}

Between April 2004 and June 2012, 362 patients with biopsy-proven primary breast cancer or extensive ductal carcinoma in situ (DCIS) necessitating mastectomy were treated according to a standardized SLN labeling protocol if the axillary lymph nodes appeared clinically inconspicuous. 9 Patients had bilateral disease and were each counted as 2 cases, resulting in a total of 371 cases. Patient records were analyzed retrospectively. Patient characteristics are listed in table 1. Patients were excluded from the analysis if a protocol violation was evident (obvious mistake in tracer preparation, failure of surgeon to describe adequate measures to retrieve SLN) from the records. In Germany, SLNB is defined as the method of choice in a clinically inconspicuous axilla [11], but no national guidelines exist on the exact labeling protocol, and since patients were not inconvenienced by the retrospective analysis, an ethics committee approval was not deemed necessary. Follow-up was warranted by scheduled annual contacts with the patients' primary physicians.

\section{Labeling Technique}

Patients were positioned with an inclination of about $30^{\circ}$ to the contralateral side of the affected breast. A depot of the radioactive tracer, either $99 \mathrm{~m} \mathrm{Tc}$ human serum albumin nanocolloid $\left(\right.$ Nanocoll $^{\circledR}$, GE Healthcare Buchler, Braunschweig, Germany) or colloidal $99 \mathrm{~m}$ Tc rhenium sulfide (Nanocis ${ }^{\circledR}$, Cis bio, Berlin, Germany) was slowly injected subepidermally at the periareolar margin of the affected quadrant.

During injection, care was taken to keep the tip of the needle visible through the skin. In a single dose, about $160 \mathrm{MBq}$ was administered in a 1.0 -ml volume, creating a wheal. After the injection, the depot was massaged until the wheal had dissolved, and a short lymph drainage of about $2 \mathrm{~min}$ in the direction of the axilla and the sternum was performed to enhance lymphatic transport of the tracer (fig. 1). 


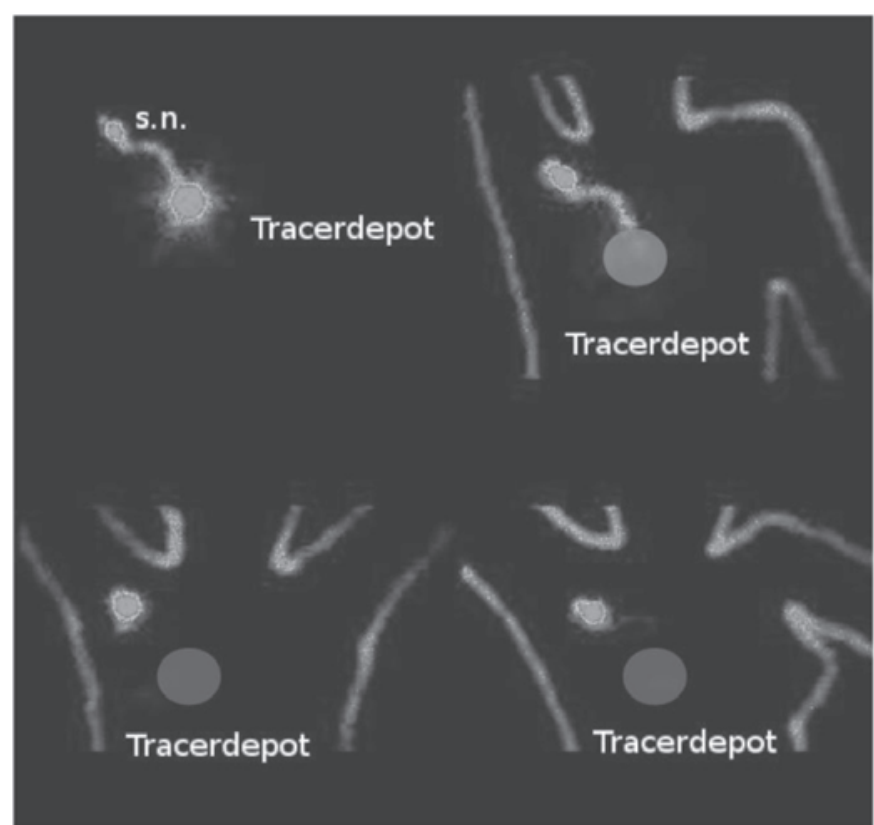

Fig. 2. Sample scintigram with body contouring in different projections.

A scintigram of the upper body was then taken using a digital singlehead SPECT camera system, with a general-purpose collimator (GKS 4, Gaede Medizinsysteme, Freiburg, Germany) and a detection parameter of 256. Subsequently, another scintigram was taken in which the tracer depot was shielded with a lead sheet. Body contours were made visible using a Tc 99m source (fig. 2). Injections and scintigrams were always performed by 1 of 2 radiologists, the senior radiologist being board certified in nuclear medicine. All operations were performed by 1 of 3 fully trained breast cancer surgeons.

The following day, 1 or more SLNs were detected intraoperatively using a hand-held probe (SI Handheld Gamma Finder, Silicon Instruments, Berlin, Germany). If a fresh frozen section of at least 1 of these nodes was positive for tumor, or if suspicious nodes were found upon surgery, formal dissection of levels 1 and 2 of the axilla (ALND) was carried out. In all, SLNB only was performed in 272 cases; in 99 cases, a formal ALND was deemed necessary.

The protocol for pathology work-up followed the German national guidelines [12] and was designed to detect all metastases of $\geq 2-m m$ diameter. Hematoxylin and eosin staining and light microscopy was performed on 2-3 serial sections spaced at $100-500 \mu \mathrm{m}$. In a subset of 276 patients, SLNs were examined on fresh frozen sections.

\section{Results}

\section{Scintigram and Pick-up Rate}

The median time to detection by scintigram was 3 min (range 1-45 min). Figure 2 shows a sample scintigram. At least 1 labeled node was identified intraoperatively in 369 of the 371 cases (range 1-9 nodes, median 2).

\section{Additional Lymph Nodes Available for Evaluation}

During SLNB, a median of 2 nodes were removed (range 1-9). In 153 of 278 cases with negative SLNs, additional unlabeled nodes that were removed incidentally during this procedure were sent to pathology in a separate specimen container ('sleepers' as opposed to 'sentinels'). A formal ALND was performed in 99 cases; 72 had a positive SLN and 25 had clinically suspicious nodes intraoperatively. In 2 cases ALND had to be performed as rescue procedure because the SLN could not be identified. During this procedure, 16 lymph nodes were removed (median, range 5-49).

\section{False-Negative Rate}

Among the 153 cases with available 'sleepers', 3 nodes were tumor positive, leading to an accurate false-negative rate of $2 \%$. Applying the usual definition [13] of false-negative results to all 91 node-positive patients in this series would yield a rate of $3.3 \%$.

In 1 case of bilateral cancer (pT2 pN1a(1/6 sn) left and pT1c $\mathrm{pN} 1 \mathrm{a}(1 / 8 \mathrm{sn})$ right), the SLN on the left-hand side was negative, although a non-labeled node that was incidentally removed was metastatic. In another case (pT2 pN1a (1/8)) treated by mastectomy for suspected bifocal breast cancer, the affected lymph node was located within the breast and was preoperatively interpreted as the second tumor. A third case (pT2, pN2a (4/16) had 2 negative SLNs, 2 positive 'sleepers' and 2 more positive nodes at formal ALND.

\section{Follow-up}

After a median period of 69.5 months (range 1.7-115.8 months), 213 of $255(83.5 \%)$ patients with a negative SLN were available for follow-up and none of them had an axillary recurrence. 1 patient developed an infraclavicular lymph node metastasis after 18.4 months.

\section{Discussion}

\section{Background Considerations}

The injection procedure is based on the method of indirect lymphangiography $[14,15]$. By creating an intradermal wheal, the tracer is driven into the initial lymph vessels by a high-pressure gradient. As shown in animal experiments with a similar injection technique, the excessive increase in tissue pressure facilitates uptake of the tracer into the initial lymph vessels through widening of open junctions and an increase in lymph vessel diameter [16]. Active transport in lymph vessels is very slow, with contraction frequencies between 6 and 10/min, but can be doubled by manual lymphatic drainage [17]. Therefore, in addition to the injection, we used a standard lymph drainage technique that has been shown to increase lymph transport into the nodes [18].

\section{Detection and Pick-up Rate}

Median time to detection was $3 \mathrm{~min}$ (range 3-45 min). This is comparable to the data reported by Sadeghi et al. [19]. Although not practiced in our routine, labeling immediately prior to surgery is possible. The pick-up rate achieved with our regime using radioactive tracer alone was $99.5 \%$. This is superior to the $97.4 \%$ observed in the series of Chagpar et al. [7] and to the $96.9 \%$ in the NSABP B32 trial [10]. 


\section{False-Negative Rate}

If an SLN is found to be negative while other lymph nodes are in fact affected, inadequate treatment with a risk of suboptimal outcome may occur. The results achieved with our regimen compare well to those of previously published reports using a combination of dye and radioactivity. The NSABP B-32 project, which included 4,994 patients, reported a false-negative rate of $9.5 \%$ [10], while Veronesi et al. [3] reported 8 false-negative SN in 259 patients with a consecutive complete axillary dissection.

Discounting the case in which an affected intramammary lymph node was preoperatively thought to be a second primary tumor and thus removed at mastectomy, in our series only 2 of 153 relevant cases demonstrated an affected non-labeled node even though the SLN was disease free. Using the standard definition [13], our false-negative rate of $3.3 \%$ compares favorably despite the omission of dye injections.

\section{Radioactive With or Without Additional Dye Labeling}

In an extensive literature search including publications from 1993 to 2015, we found only 3 series reporting results for radiotracer labeling only. Ban et al. [20] reported on 328 patients, with a retrieval rate of $100 \%$ and a false-negative rate of $10.8 \%$. Bines et al. [21] examined 208 patients and retrieved $93.8 \%$ of labeled nodes; however, they did not report a false-negative rate. Again, our results compare favorably.

Recently, O'Reilly et al. [22] pointed out that 'most studiesdemonstrating an improvement in identification rates and lower false-negative rates with combined localization techniques were early in the learning curve of individual surgeons' and that it was subsequently found, in a randomized prospective trial, that the addition of dye labeling did not lead to superior results and should, therefore, no longer be a part of routine practice.
Patent blue, which is commonly used for lymphatic mapping, leads to severe and typically prolonged anaphylactic reactions in $1-3 \%$ of patients $[23,24]$. Methylene blue, which is at present not commercially available in most European countries, is safer but may lead to temporary tattooing at the injection site and is possibly neurotoxic $[25,26]$.

\section{Axillary Recurrence}

In our series, 255 patients underwent SLNB only; of these, 213 (83.5\%) were available for follow-up. During the comparatively long follow-up period (median 69.5 months, range 1.7115.8 months) no axillary and only 1 subclavicular recurrence were seen. Smidt et al. [27] reported 2 recurrences among 439 patients after 26 months in their own series and, from an extensive literature search, 8 in 3,184 after 21 months. Takei et al. [28] made a distinction in local recurrence between axillary metastases, which they found in $0.4 \%$ of 1,062 patients, and other local disease including infraclavicular recurrence, which they saw in $2.4 \%$. The follow-up period in their series was 36 months. Again, our regime performed better.

\section{Conclusion}

The combined technique of periareolar intradermal radioactive tracer injection and subsequent manual lymphatic drainage is easy to perform and gives excellent and quick results. Our results add to the body of evidence that additional dye labeling is unnecessary when following a standardized radioactive labeling regime.

\section{Disclosure Statement}

All authors have approved of the final version to be submitted and are not aware of any conflict of interest.

\section{References}

1 Goldhirsch A, Winer EP, Coates AS, et al.: Personaliz ing the treatment of women with early breast cancer: Highlights of the St Gallen International Expert Consensus on the Primary Therapy of Early Breast Cancer 2013. Ann Oncol 2013;24:2206-2223.

- 2 Armer JM, Fu MR, Wainstock JM, et al.: Lymphedema following breast cancer treatment, including sentinel lymph node biopsy. Lymphology 2004;37:73-91.

3 Veronesi U, Paganelli G, Galimberti V, et al.: Sentinelnode biopsy to avoid axillary dissection in breast cancer with clinically negative lymph-nodes. Lancet 1997; 349:1864-1867.

4 Wernicke AG, Goodman RL, Turner BC, et al.: A 10 year follow-up of treatment outcomes in patients with early stage breast cancer and clinically negative axillary nodes treated with tangential breast irradiation following sentinel lymph node dissection or axillary clearance. Breast Cancer Res Treat 2011;125:893-902.

5 American College of Breast Surgeons https://www. breastsurgeons.org/statements/2010-Nov-05_Guidelines_on_Performing_SLN.pdf. Last accessed on 29 June 2015.
6 Lyman GH, Giuliano AE, Somerfield MR, et al.: American Society of Clinical Oncology American Society of Clinical Oncology guideline recommendations for sentinel lymph node biopsy in early-stage breast cancer. J Clin Oncol 2005;23:7703-7720.

7 Chagpar AB, Martin RCG, Chao C, et al.: Validation of subareolar and periareolar injection techniques for breast sentinel lymph node biopsy. Arch Surg 2004; 139:614-618; discussion 618-620.

8 D'Eredita G, Ferrarese F, Cecere V, et al.: Subareolar injection may be more accurate than other techniques for sentinel lymph node biopsy in breast cancer. Ann Surg Oncol 2003;10:942-947.

$\checkmark$ Yen R-F, Kuo W-H, Lien H-C, et al.: Radio-guided sentinel lymph node biopsy using periareolar injection technique for patients with early breast cancer. J Formos Med Assoc 2007; 106:44-50.

10 Krag DN, Ashikaga T, Harlow SP, et al.: Surgeon training, protocol compliance, and technical outcomes from breast cancer sentinel lymph node randomized trial. J Natl Cancer Inst 2009;101:1356-1362.

11 http://www.ago-online.de/fileadmin/downloads/ leitlinien/mamma/oktober2006/02OperationUnter OnkologischenAspekten.pdf. Last accessed on 4 June 2014.
12 Informationszentrum für Standards in der Onkologie (ISTO) Deutsche Krebsgesellschaft e.V. (Hrsg.): Interdisziplinäre S3-Leitlinie für die Diagnostik, Therapie und Nachsorge des Mammakarzinoms. Zuckschwerdt, München.

13 Nieweg OE, Estourgie SH: What is a sentinel node and what is a false-negative sentinel node? Ann Surg Oncol 2004;11(3 Suppl):169S-173S

14 Partsch H, Wenzel-Hora BI, Urbanek A: Differential diagnosis of lymphedema after indirect lymphography with Iotasul. Lymphology 1983;16:12-18.

15 Weissleder H, Weissleder R: Interstitial lymphangiography: Initial clinical experience with a dimeric nonionic contrast agent. Radiology 1989;170:371-374.

16 Castenholz A, Zöltzer H: Neue Erkenntnisse zur strukturellen Grundlage der Lymphbildung. Z Lymphol 1989;8:23-31.

17 Olszewski WL: Contractility of human leg lymphatics: Clinical implications. Scope Phlebol Lymphol 1998;4: 16-20.

18 Hutzschenreuter P, Bruemmer H, Ebberfeld K: Experimentelle und klinische Untersuchungen zur Wirkungsweise der manuellen Lymphdrainage-Therapie. Z Lymphol 1989;8:62-64. 
19 Sadeghi R, Forghani MN, Memar B, et al.: How long the lymphoscintigraphy imaging should be continued for sentinel lymphnode mapping? Ann Nucl Med 2009;23:507-510.

20 Liang MI, Carson WE: Biphasic anaphylactic reaction to blue dye during sentinel lymph node biopsy. World J Surg Oncol 2008;6:79.

21 Mertes PM, Alla F, Tréchot P, et al.: Anaphylaxis during anesthesia in France: An 8-year national survey. J Allergy Clin Immunol 2011;128:366-373.

22 Varghese P, Abdel-Rahman AT, Akberali S, et al. Methylene blue dye-a safe and effective alternative for sentinel lymph node localization. Breast J 2008;14:6167.
23 Rowley M, Riutort K, Shapiro D, et al.: Methylene blue-associated serotonin syndrome: a 'green' encephalopathy after parathyroidectomy. Neurocrit Care 2009;11:88-93.

24 Ban EJ, Lee JS, Koo JS, et al.: How many sentinel lymph nodes are enough for accurate axillary staging in T1-2 breast cancer? J Breast Cancer 2011;14:296300.

25 Bines S, Kopkash K, Ali A, et al.: The use of radioisotope combined with isosulfan Blue dye is not superior to radioisotope alone for the identification of sentinel lymph nodes in patients with breast cancer. Surgery 2008:144:606-609.
26 O’Reilly EA, Prichard RS, Al Azawi D, et al.: The value of isosulfan blue dye in addition to isotope scanning in the identification of the sentinel lymph node in breast cancer patients with a positive lymphoscintigraphy: A randomized controlled trial (ISRCTN98849733). Ann Surg 2015;262:243-248.

27 Smidt ML, Janssen CM, Kuster DM, et al.: Axillary recurrence after a negative sentinel node biopsy for breast cancer: Incidence and clinical significance. Ann Surg Oncol 2005;12:29-33.

28 Takei H, Suemasu K, Kurosumi M, et al.: Recurrence after sentinel lymph node biopsy with or without axillary lymph node dissection in patients with breast cancer. Breast Cancer 2007;14:16-24. 